\title{
TRANSPORTE DE PACIENTES POR MACAS HOSPITALARES: UMA ANÁLISE METODOLÓGICA
}

RODRIGUES, Yago W., Me.; programa de pós-graduação em Design - UFPR yagowr5@gmail.com

OKIMOTO, Maria Lucia Leite Ribeiro, Dra. Eng.; programa de pós-graduação em Design - UFPR lucia.demec@ufpr.br

HEEMANN, Adriano, Dr. do programa de pós-graduação em Design - UFPR adriano.heemann@ufpr.br

Resumo: Este artigo apresenta um levantamento bibliográfico sobre os aspectos ergonômicos do transporte de pacientes em hospitais no Brasil. A metodologia empregada foi uma revisão bibliográfica sistemática para mapear os principais estudos sobre o tema, articulando evidências empíricas produzidas sobre as características da avaliação ergonômica do transporte de pacientes por macas hospitalares. A análise descreve os aspectos de avaliação através da metodologia utilizada pelos autores e problematiza a grande ocorrência da abordagem observativa e postural. A pesquisa realça os problemas enfrentados pelos trabalhadores da saúde e discute as novas exigências de pesquisa na área da ergonomia e usabilidade para o transporte com macas hospitalares.

Palavras-chave: Ergonomia hospitalar, avaliação ergonômica, transporte de paciente, maca hospitalar.

Abstract: This paper presents a literature survey on the ergonomic aspects of patients transport in hospitals in Brazil. The methodology used was a literature systematic review to map the major studies on the topic, articulating produced empirical evidence on the characteristics of the ergonomic evaluation of patient transport for hospital beds. The analysis describes the evaluation's aspects through the methodology used by the authors and discusses the wide occurrence of observation and postural approach. The research highlights the problems faced by health care providers and discusses the changing demands of research in ergonomics and usability for transport to hospital beds.

Key-words: Hospital ergonomics, ergonomic evaluation, patient transport, hospital bed. 


\section{INTRODUÇÃO}

O campo de atuação da ergonomia hospitalar, segundo Maria e Cardoso (1998), tem por objetivo o melhoramento das condições de trabalho, conforto e segurança dos trabalhadores e dos pacientes na área da saúde, envolvendo e adaptando o ambiente ao trabalhador através de equipamentos seguros. Desta maneira, a ergonomia hospitalar concentra-se na área da ergonomia física e aborda as caraterísticas humanas, os aspectos anatômicos, antropométricos, fisiológico e biomecânico nas suas relações com atividade física. Também inclui tópicos relacionados com a postura no trabalho, manipulação de equipamentos, movimentos repetitivos, segurança e a saúde ocupacional (International Ergonomic Association, 2000).

Estryn-Behar (2001) descreve que apenas recentemente a aplicação da ergonomia em hospitais tornou-se conhecida e sua difusão ainda está muito limitada. Ademais, é observado uma grande quantidade de publicações cujo objeto é a ergonomia e, com raras exceções, existem textos relacionados às metodologias de análise ergonômica hospitalar, que frequentemente se encontram dispersas (LIMA, 2004). Poucas pesquisas abordam a análise do produto com uma visão do paciente transportado envolvendo teste de simulação com cenários reais, aplicando uma metodologia de movimento dinâmico, na qual, avalia através da atividade de empurrar o equipamento e o esforço da real atividade.

De acordo com a revisão de Bernardes e Moro (2011) o tema de transferência de pacientes é uma questão carente em publicações na literatura científica nacional, principalmente na área preventiva, ainda mais, no que diz respeito ao transporte de pacientes através da maca hospitalar.

No Brasil, em relação aos fatores de risco relacionados especificamente com as atividades profissionais no hospital, um dos mais citados é o transporte de paciente por macas, pois o transportador realiza a atividade repetitivamente com elevadas cargas (ALEXANDRE, 1998).

Para Looze et al. (1994, p. 427-431) o meio mais eficiente para a redução da sobrecarga física é através do redesenho de equipamentos. E para isto, são necessárias metodologias para coleta de requisitos no projeto de design. Logo, para a aplicação da ergonomia é necessário avaliações mais coesas do produto, através de teste de usabilidade da atividade envolvendo o enfermeiro- maca -ambiente.

Diante do contexto apresentado, o objetivo desta pesquisa é explorar os aspectos ergonômicos de avaliação do transporte de paciente pelas c macas hospitalares, através de uma síntese de obras. Assim, através de uma revisão bibliográfica é possível identificar a situação sobre ergonomia para a maca de transporte, como os aspectos ergonômicos são avaliados e que método foi utilizado em âmbito nacional. 


\section{METODOLOGIA}

A metodologia empregada no presente estudo foi uma revisão sistemática bibliográfica (RBS) articulando evidências empíricas produzidas sobre aspectos ergonômicos das macas, que, possibilita o embasamento teórico para dar continuidade ao processo. A exploração é compreendida num processo de coletar, assimilar, analisar, sintetizar e avaliar um conjunto de artigos científicos a fim de criar uma estrutura completa (CONFORTO et al. adaptado de LEVY; ELLIS, 2006).

A revisão é realizada através de pesquisas de artigos por pares a partir do ano 2000 na área de ergonomia hospitalar, com a finalidade de mapear de forma atualizada o que se produziu em âmbito nacional em periódicos. Para coleta de dados foi realizado buscas no mês de março de 2014 com os termos: "ergonomia AND hospital AND transporte AND maca" em todos os campos dos artigos nas bases de dados eletrônicas (Google Acadêmico, Periódicos da CAPES, Science Direct e Scopus). Assim, foram constatados 521 artigos no total, que ao passar do processo foram filtrados por título, resumo, objetivos e método ergonômico, chegando a 10 artigos analisados.

Após a apresentação dos resultados, o presente artigo discute e lista os principais autores e conceitos considerados essenciais ao transporte de pacientes em hospitais no Brasil, com finalidade de estruturar os objetivos e métodos da pesquisa. Porém, sem fazer comparações entre os autores, apenas focado na problemática da análise da observação ergonômica quando envolve equipamentos dinâmicos, neste caso, a maca de transporte de pacientes.

\section{RESULTADOS}

Os problemas de sáude desenvolvidos pelos trabalhadores por conta do transporte de macas é muito comum devido ao alto esforço e repetição do movimento durante a tarefa. Conhecido como lesões por esforços repetitivos (LER) é apontado como uma das causas primárias para o desenvolvimento de distúrbios osteomusculares relacionados ao trabalho (DORT) (NELSON; BAPTISTE, 2006; KARAHAN et al., 2009.)

Segundo Botha; Bridger, (1998), esses casos se alastraram nas últimas décadas o que representa um sério dilema de saúde pública, como a incapacidade ocupacional, produzindo custos expressivos em tratamento e indenizações (Ministério da Saúde, 2003; MAGNAGO et al., 2010), pois os trabalhadores são susceptíveis a lesões pelo fato de terem de movimentar e transportar pacientes regularmente, o que representa um evento acumulativo, que predispõe, sobretudo de dores vertebrais (STORDEUR et al., 2001; CABOOR et al., 2000).

Percebe-se que a Análise Ergonômica do Trabalho (AET), em unidades de saúde, tem permitido o reconhecimento das variáveis humanas e ambientais, gerenciada com maior ou menor sucesso a eficácia num sistema complexo, envolvendo o trabalhador (FLORENTINO et al., 2010). No entanto, muitos autores abordam a avaliação ergonômica através de uma avaliação observatória e postural, sem um aprofundamento da ergonomia física dinâmica na análise da atividade. Esse aprofundamento dar-se-ia a partir de uma análise biomecânica das exigências físicas dos profissionais de saúde (CARAYON, 2010), centrada numa visão específica, por 
exemplo, da área muscular, o peso da carga física ou avaliação de simuladores da atividade com o equipamento.

Determinados fatores de risco ergonômicos são analisados na literatura em hospitais sobre o transporte de pacientes, estes, são causados principalmente pela falta de equipamentos auxiliares, desníveis de altura entre a cama hospitalar e a maca de transporte, macas de difícil movimentação e a falta de travas nas rodas. (OWEN, 2000; CÉLIA; ALEXANDRE, 2004). Foi percebido que muitos problemas também são de origem do layout do posto de trabalho (posicionamento dos corredores), questões de logística (materiais) e de manutenção (MARZIALE e ROBAZZI, 2000.)

Assim, existem diversos métodos capazes de efetuar a análise ergonômica de transporte de pacientes. Será apresentado a seguir o que já foi realizado sobre o tema, através da abordagem dos principais autores e sua metodologia. Conforme a classificação de Gil (1991), o quadro 1 apresenta os autores, ano de publicação, objetivo e a metodologia utilizada em ordem cronológica.

Constata-se a partir do levantamento que com exceção de um artigo (CORRÊA et al., 2004) o restante foi publicado por pesquisadores da área da saúde em periódicos específicos de seus campos disciplinares e, em sua totalidade aplicaram um estudo de caso como metodologia de avaliação:

Quadro 1 - Lista de autores relevantes nacionais.

\begin{tabular}{|c|c|c|}
\hline Autores & Objetivo & Metodologia \\
\hline $\begin{array}{l}\text { ZANON e } \\
\text { MARZIALE (2000) }\end{array}$ & $\begin{array}{l}\text { Analisar as posturas assumidas pelos } \\
\text { trabalhadores de enfermagem na } \\
\text { movimentação de pacientes acamados. }\end{array}$ & $\begin{array}{l}\text { Estudo de caso, observação } \\
\text { direta: gravação de vídeo da } \\
\text { atividade. }\end{array}$ \\
\hline ROSSI et al. (2001) & $\begin{array}{l}\text { Avaliar aspectos ergonômicos na } \\
\text { transferência de pacientes com a utilização } \\
\text { de maca e cadeira de rodas. }\end{array}$ & $\begin{array}{l}\text { Estudo de caso, observação e } \\
\text { questionário quali-quantitativo: } \\
\text { dados gerais, específicos e sobre } \\
\text { o paciente. }\end{array}$ \\
\hline $\begin{array}{l}\text { PARADA et al. } \\
(2002)\end{array}$ & $\begin{array}{l}\text { Investigar determinadas características da } \\
\text { ocorrência de acidentes do trabalho } \\
\text { relacionados com a coluna vertebral em } \\
\text { trabalhadores de enfermagem de um } \\
\text { hospital. }\end{array}$ & $\begin{array}{l}\text { Estudo de caso, entrevista } \\
\text { estruturada com o uso de um } \\
\text { formulário com perguntas } \\
\text { abertas e fechadas sobre } \\
\text { atividade e acidentes. }\end{array}$ \\
\hline $\begin{array}{l}\text { GALLASCH e } \\
\text { ALEXANDRE (2003) }\end{array}$ & $\begin{array}{l}\text { Mapear os riscos ergonômicos durante os } \\
\text { procedimentos nas unidades de um } \\
\text { hospital. }\end{array}$ & $\begin{array}{l}\text { Estudo de caso, questionário: } \\
\text { escala de avaliação dos riscos na } \\
\text { movimentação e transferência do } \\
\text { paciente. }\end{array}$ \\
\hline $\begin{array}{l}\text { BARROS e } \\
\text { ALEXANDRE (2003) }\end{array}$ & $\begin{array}{l}\text { Adaptar culturalmente o conteúdo geral do } \\
\text { Questionário Nórdico para o português e } \\
\text { avaliar a confiabilidade de sua versão } \\
\text { brasileira. }\end{array}$ & $\begin{array}{l}\text { Estudo de caso, questionário } \\
\text { nórdico adaptado (teste e } \\
\text { reteste) pela análise da } \\
\text { concordância Kappa. }\end{array}$ \\
\hline $\begin{array}{l}\text { CÉLIA e } \\
\text { ALEXANDRE (2004) }\end{array}$ & $\begin{array}{l}\text { Identificar as atividades ocupacionais que } \\
\text { exigem mais esforços e verificar os sintomas } \\
\text { musculoesqueléticos em trabalhadores. }\end{array}$ & $\begin{array}{l}\text { Estudo de caso - entrevista: } \\
\text { escala de Percepção Subjetiva do } \\
\text { Esforço (RPE) ou escala BORG. }\end{array}$ \\
\hline $\begin{array}{l}\text { CORRÊA et al. } \\
(2004)\end{array}$ & $\begin{array}{l}\text { Apresentar os resultados de uma } \\
\text { abordagem ergonômica preliminar das } \\
\text { condições dos profissionais de enfermagem, } \\
\text { desenvolvida a partir do levantamento dos } \\
\text { principais problemas biomecânicos. }\end{array}$ & $\begin{array}{l}\text { Estudo de caso, questionário: } \\
\text { nível de desconforto do Diagrama } \\
\text { de Corllet e Manenica e avaliação } \\
\text { do nível de conforto/desconforto } \\
\text { da atividade. }\end{array}$ \\
\hline
\end{tabular}




\begin{tabular}{|c|c|c|}
\hline ALEXANDRE (2007) & $\begin{array}{l}\text { Descrever pontos básicos das principais } \\
\text { estratégias de prevenção e controle dos } \\
\text { sintomas osteomusculares, em } \\
\text { trabalhadores da área de saúde. }\end{array}$ & $\begin{array}{l}\text { Revisão de artigos internacionais, } \\
\text { demostra com imagens as } \\
\text { posturas adequadas. }\end{array}$ \\
\hline $\begin{array}{l}\text { FRONZA e TEIXEIRA } \\
(2010)\end{array}$ & $\begin{array}{l}\text { Pesquisar sobre a percepção do } \\
\text { trabalhador quanto às suas condições } \\
\text { dolorosas, considerando a convivência com } \\
\text { a dor e a interferência disto sobre a } \\
\text { qualidade de vida no trabalho e sua vida } \\
\text { social. }\end{array}$ & $\begin{array}{l}\text { Estudo de caso, questionário } \\
\text { Nórdico adaptado e questionário } \\
\text { SF-36: Uma abordagem } \\
\text { qualitativa e quantitativa, com } \\
\text { característica observacional. }\end{array}$ \\
\hline $\begin{array}{l}\text { RODRIGUES et al. } \\
\text { (2013) }\end{array}$ & $\begin{array}{l}\text { Desenvolver uma análise das condições } \\
\text { ergonômicas dos profissionais de } \\
\text { enfermagem em um hospital }\end{array}$ & $\begin{array}{l}\text { Estudo de caso, questionário: } \\
\text { Nível de desconforto Diagrama de } \\
\text { Corllet e Manenica. }\end{array}$ \\
\hline
\end{tabular}

Fonte: Elaborado pelo autor, com base na pesquisa realizada.

\section{DISCUSSÃO}

A discussão sobre as pesquisas relevantes para o trabalho está direcionada na metodologia abordada pelos autores para avaliar as macas de transporte. A pesquisa direcionou maior destaque aos métodos ergonômicos e não aos estatísticos.

Segundo Zanon e Marziale (2000) a pesquisa é de caráter quali-quantitativo descritivo, aplicada através da metodologia de questionário e observação de filmagens, envolvem desde a idade, sexo e tempo do trabalhador no hospital até características de como realiza determinada tarefa, com opiniões dos enfermeiros e posições posturais, assim, categorizaram fatores que facilitam e dificultam o transporte de pacientes. A metodologia serviu para coletar os problemas de origens dos DORT, como o espaço físico inadequado, grande carga física dependida, equipamentos danificados e entre outros aspectos. Porém, não é uma pesquisa específica sobre transporte de pacientes por macas, no sentido horizontal, ou aborda metodologias de movimento dinâmico do equipamento.

No estudo de Rossi et al. (2001), é verificável uma avaliação através de observação quantitativa dos aspectos envolvendo os tipos de transferência dos pacientes com um estudo de caso. Discutem os problemas e a falta de equipamentos, os tipos de paciente, a falta de preparo e má postura dos trabalhadores. Assim, levantam porcentagens a cerca da situação ergonômica do hospital numa forma mais holística, não envolvendo características especifica da carga ou analise ergonômica dos equipamentos.

Parada et al. (2002) realizaram a pesquisa no hospital através de três partes: identificação dos trabalhadores; questões relacionadas com o acidente; questões relacionadas com os acidentes que ocorreram durante a movimentação, transferência ou transporte dos pacientes. Constataram que o ambiente e o paciente interferem nas causas, principalmente o piso escorregadio (a segunda maior ocorrência de acidente foi no corredor externo da unidade) e o peso excessivo da carga (paciente).

Gallasch et al. (2003) aplicaram uma avaliação das condições físicas de preparo do paciente, do ambiente e dos equipamentos da equipe utilizando o instrumento denominado escala de avaliação do risco na movimentação e transferência de paciente (RADOVANOVIC; ALEXANDRE, 2002). Isso permite avaliar aspectos desde o peso, 
altura, tipo de transferência, mobilidade na maca Verificando qual o grau de risco oferecido pelas unidades de internação de um hospital (pouco, médio e alto risco). Entretanto, não era o enfoque da pesquisa a atividade de transporte de pacientes pelas macas com a ajuda do enfermeiro (empurrando a maca) e sim, de todos os problemas de transportes de pacientes do hospital (âmbito organizacional).

Em 2003, Barros e Alexandre, adaptam o questionário nórdico que é uma metodologia reconhecida mundialmente, pois avalia problemas musculoesqueléticos para o português testando-o em estudantes de enfermagem e professores (teste e reteste), para averiguar o uso desta metodologia, formando uma análise quantitativa na área da saúde ocupacional do trabalhador. Após os testes utilizaram o coeficiente de Kappa para comparar os resultados, existindo mais convergências que divergências na análise.

O questionário nórdico contém uma figura com nove regiões anatômicas do corpo, representadas nas vistas anterior e posterior do pescoço aos pés. As questões relacionam-se com cada área anatômica, associando-se as dores a determinados fatores, como grande exigência muscular nas atividades de vida diária, a prática de outra atividade profissional, a realização de exercícios físicos, as dores relatadas durante o trabalho, as horas trabalhadas e também a idade e sexo do trabalhador. $\mathrm{Na}$ adaptação de Barros e Alexandre (2003) encontramos uma divisão feita em nove regiões anatômicas ( 3 de membros superiores, 3 de membros inferiores e 3 de tronco) que compreendem questões relativas à presença de dores osteomusculares no corpo nos últimos 12 meses e nos últimos 7 dias. Essa abordagem é muito útil para visualizar questões de causas ocupacionais, de forma específica do músculo ou membro.

Outro artigo semelhante de Célia e Alexandre (2004) utilizam a derivação do questionário nórdico e também aplicam a escala PSE (percepção subjetiva de esforço) conhecida como escala BORG, que permite avaliar as atividades percebidas como de maior esforço para o sistema musculoesquelético em sua totalidade e suas implicações ergonômicas. Essa escala apresenta escores (mínimo esforço - máximo esforço) e avalia os níveis dos esforços que os trabalhadores sentem durante a realização da atividade, mas a pesquisa envolvia outros funcionários, como motoristas e também outras possibilidades de transporte; como por exemplo, da cadeira de rodas para maca. Segundo a pesquisa, as atividades relacionadas ao transporte de pacientes requerem muito esforço para a coluna vertebral, principalmente para o transporte da ambulância até a cama do paciente, onde é a terceira atividade que exige mais esforço para o sistema musculoesquelético e sua totalidade. Aqui, também, preocupou-se com todas as atividades de risco de qualquer funcionário do hospital em relação aos variados tipos de transportes de pacientes. Essa configuração de pesquisa apontou de forma detalhada partes do corpo humano onde são prejudicadas pelo trabalho, principalmente a região lombar.

Em 2010, Fronza e Teixeira, também utilizaram o questionário nórdico de sintomas osteomusculares em paralelo com questionário SF-36 que aborda itens referentes à percepção da saúde dos profissionais na atualidade e no ano anterior, sendo também investigada a interferência de queixas nas atividades laborativas e sociais, envolvendo saúde física e emocional.

Os autores Corrêa et al. (2004) e Rodrigues et al. (2013) identificaram o desconforto dos trabalhadores no hospital, avaliado através do diagrama de Corllet e Manenica (1980). Que indica a incidência do nível de desconforto percebido em partes 
do corpo em porcentagens, semelhante ao questionário nórdico. Eles também caracterizaram as atividades ocupacionais, conforme os outros autores. Nesta pesquisa, é notável uma especificação que avalia melhor o desconforto no corpo, pois define áreas de distribuição da tensão. Apesar de não ser uma pesquisa única sobre transporte de pacientes por macas, pois também engloba um conjunto na área de transportes de paciente.

Em paralelo ao diagrama, Corrêa et al. (2004) realizam também uma pesquisa qualitativa que demostrou o desconforto dos trabalhadores através do questionário de nível de conforto descrito por Alexandre e Rogante (2000). Esta é uma escala respondia pessoalmente sobre a atividade (subjetiva), entretanto, não especifica a atividade de empurrar o paciente pela maca hospitalar, seja no corredor, seja quando o paciente chega de ambulância.

Alexandre (2007) descreve orientações básicas dentro do referencial teórico da ergonomia para facilitar o transporte de pacientes. Demostra por imagens posturas e formas corretas de transporte e manuseio. Este, deve englobar os equipamentos, as tarefas, a equipe e o ambiente, como a orientação da atividade e manuseio do paciente que auxiliam o trabalhador de enfermagem contra dores na coluna, com uma abordagem preventiva e educativa, demostrando barreiras e soluções que precisam ser implementadas na realidade brasileira. Porém, não é relatada nenhuma atividade específica sobre o transporte de macas ou macas hospitalares, como posições ideais para empurrá-la com o paciente deitado ou sentado nesse transporte, que envolva carga, velocidade e antropometria.

De forma geral, os aspectos de avalição ergonômica servem para determinar a intensidade de constrangimentos osteomusculares, avaliar o peso da carga e as consequências causadas pelas más posturas mantidas durante a realização do trabalho, assim, sugere possíveis soluções visando alcançar o bem-estar, visualizando através de gráficos, tabelas, ilustrações (DINIZ; MORAES, 2001). Servem também para qualificar as observações da AET na análise real da atividade do transporte.

Todos os autores preocuparam-se com os fatores do trabalhador, com sua postura e forma de prevenir acidentes no transporte de pacientes, identificando causas e problemas. Para isso, utilizaram metodologias que abordam a relação entre o servidor e os pacientes, indo ao encontro de uma abordagem da ergonomia ocupacional que, aliás, não se caracteriza pela análise da estrutura do produto e características específicas da maca Chegam, entretanto, a tecer algumas ponderações, nada aprofundado, a respeito dos problemas nas rodas das macas, ferrugem, tamanho pequeno da maca, falta de manutenção e desgastes. Vale destacar que as pesquisas realizadas não tinham a intenção de abordar de forma específica uma análise restrita à movimentação. Logo, não simulam aspectos ideais para o transporte por maca, ainda mais em situações de emergências, onde o trabalhador, o paciente, o equipamento, os obstáculos e o tempo, são fortes fatores que determinam a avaliação da ergonômica física de forma holística.

Ademais, na ergonomia a observação é o centro dos métodos para se compreender as reais condições de trabalho, as atividades efetivas de operadores e seus resultados sobre a saúde e a produção, conforme abordam Guérin et al. (2004). Além disso, Volpi (s.d., p.1) descreve que a análise deve ser um trabalho minucioso que requer extrema paciência, dedicação, além de profundo conhecimento técnico, pois mencionar se uma situação é apenas satisfatória ou insatisfatória sem propor soluções, 
não condiz com a profundidade do problema. Contudo, a desvantagem, segundo Couto (1995), é que a análise minuciosa produz poucos resultados práticos, porém a visão geral do processo de trabalho, observando apenas por cima as situações, traz grandes problemas. A análise macroscópica é a visão geral do trabalho, sendo facilmente percebível pelo observador (como posturas inadequadas, alturas desproporcionais e etc). Na análise microscópica, por outro lado, encontramos os pormenores envolvidos, abrangendo questões relacionadas ao trabalho manual e ao método de trabalho, com foco em detalhes "mínimos" (COUTO, 1995).

Por fim, conforme aborda lida (2005), a ergonomia demonstra de maneira qualificada as interações das pessoas com a tecnologia, a organização e o ambiente, intervindo nos projetos para melhorar, de forma integrada, e não dissociada, a segurança, o conforto, o bem-estar e a eficácia das atividades humanas. A partir desse argumento, pode-se pensar a avalição da ergonomia física de forma específica aplicada no produto, que envolve todas essas variáveis e também é uma forma de qualificação para coleta e análise de dados.

\section{CONCLUSÃO}

A pesquisa centrou-se no reconhecimento dos aspectos avaliativos ergonômicos do transporte de pacientes por maca em hospitais no Brasil. Foi identificado uma grande ocorrência da abordagem médica, que compreende uma visão que se relaciona com o design. Porém existem diferenças em alguns aspectos, porque as pesquisas abordam o saber ergonômico pelo ponto de vista da medicina, como parâmetros preventivos e ocupacionais, e não uma visão da ergonomia do produto com a abordagem específica do design, mesmo coexistindo características em comum, já que se trata de um objeto de pesquisa interdisciplinar.

O setor hospitalar é um exemplo no qual as condições ocupacionais geram demanda ergonômica, possivelmente em decorrência da interface ocupacional com equipamentos tecnológicos mal projetados e os problemas relacionados à repetição da atividade no transporte pelas maca de transporte. 0 transporte de enfermos pela maca carece de avaliação de carga no uso horizontal da força, bem como análises do equipamento em simuladores e plataformas 3D com metodologias para movimentos dinâmicos.

Como foi evidenciado nos resultados e na discussão deste estudo, há que se investigar mais, de forma prescrita e sistematizada, o entorno do problema do transporte de pacientes por maca, principalmente a relação do usuário e do trabalhador, identificando e propondo soluções na área do design para a área hospitalar de transporte Essas soluções devem, necessariamente, buscar uma maior interação entre trabalhador, maca, paciente e ambiente.

Por fim, através do mapeamento bibliográfico, foi possível compreender o dimensionamento do assunto de forma mais detalhada e profunda, identificando as pesquisas mais significativas produzidas no Brasil. Esse mapeamento contribuiu para o entendimento das características de avaliação da ergonomia, na visualização de lacunas existentes e futuras pesquisas centradas na área do design. 


\section{AGRADECIMENTOS}

Agradecemos ao Conselho Nacional de Desenvolvimento Científico e Tecnológico (CNPq), a Coordenação de Aperfeiçoamento de Pessoal de Nível Superior (CAPES) e ao Programa de Pós-Graduação em Design (PPGDesign).

\section{REFERÊNCIAS}

ALEXANDRE, N.M.C. Aspectos ergonômicos relacionados com $\mathbf{o}$ ambiente e equipamentos hospitalares. Rev. latino-am. enfermagem - Ribeirão Preto, v. 6 - n., p. 103-109, 1998.

ALEXANDRE, N.M.C.; ROGANTE, M. M. Movimentação e transferência de pacientes: aspectos posturais e ergonômicos. Revista da Escola de Enfermagem USP, 34 (2), 165$173,2000$.

BARROS, E.N.C.; ALEXANDRE N.M.C. Cross-cultural adaptation of Nordic musculoskeletal questionnaire. International Nursing Review, Geneva. June; 50(2):101-8, 2003.

BERNARDES, J.M.; MORO, A. R. P. Movimentação e transferência de pacientes: uma análise da produção científica nacional. XXI Encontro Nacional De Engenharia De Produção- ENEGEP. Belo Horizonte: outubro de 2011.

BOTHA, W.E.; BRIDGER R.S. Anthropometric variability, equipament usability and musculoskeletal pain in a group of nurses in the Western Cape. Applied Ergonomics, 229: 481-90, 1998.

CABOOR, D. E. et al. Implications of an adjustable bed height during standart nursing tasks on spinal motion, perceived exertion and muscular activity. Ergonomics. 43(10): 177-80, 2000.

CARAYON, P. Human factors in patient safety as an innovation. Applied Ergonomics, 41:657-65, 2010.

CÉLIA, R.C.R.S.; ALEXANDRE, N.M.C. Aspectos ergonômicos e sintomas osteomusculares em um setor de transporte de pacientes. Rev Gaúcha Enferm, Porto Alegre (RS), v. 25 (1), n. 1, p. 33-43, 2004.

CONFORTO, E. C. et al. Roteiro para Revisão Bibliográfica Sistemática: aplicação no desenvolvimento de produtos e gerenciamento de projetos. Congresso Brasileiro de Gestão de Desenvolvimento de Produto - CBGDP 2011. Porto Alegre: Instituto de Gestão de Desenvolvimento de Produto. 2011. 
CORLLET, E.N.; MANENICA, I. The effects and measurement of working postures. Applied Ergonomics. 11 (01): 7-16, 1980.

CORRÊA, J. A. et al. Os problemas ocupacionais dos profissionais de enfermagem e a necessidade em aplicar design ergonômico nos equipamentos médico-hospitalar. Revista Assentamentos Humanos, Marília, v6, nำ1, pag. 75 - 82, 2004.

COUTO, H. A. Ergonomia aplicada ao trabalho; o maual técnico da máquina humana. 2v. Belo Horizonte. Ergo, 1995.

DINIZ, R. L.; MORAES, A. Aplicação da intervenção ergonomizadora: o caso do trabalho em cirurgias eletivas gerais. Ação Ergonômica, v. 1, $n^{\circ} 2$, n. 2000, p. 46-61, 2001.

ESTRYN-BEHAR, M.R. Hospital Ergonomia: Uma Revisão. Ergonomia e Saúde. Enciclopédia de Saúde e Segurança Ocupacional, Jeanne Mager Stellman, Editor-in Chief. Organização Internacional do Trabalho, Genebra, 2011.

FLORENTINO, S. et al. Ergonomia hospitalar e segurança do doente: mais convergências que divergências. Rev. Port Saúde Pública, v. Temat(10):, n. 10, p. 5873, 2010.

GALLASCH, C. H; ALEXANDRE, N.M.C. Avaliação dos Riscos Ergonômicos Durante a Movimentação e Transporte de Pacientes em Diferentes Unidades Hospitalres. Rev.Enferm. UERJ, v. 11;2, p. 52-60, 2003.

GIL, A. C. Como elaborar projetos de pesquisa. São Paulo: Atlas, 1991.

GUÉRIN, F. et al. Compreender o trabalho para transformá-lo: a prática da ergonomia. Edgard Blucher: Fundação Vanzolini, São Paulo, 2004.

IEA. International Ergonomics Association: Triennial report. Santa Monica, CA: IEA Press, 2000.

IIDA, Itiro. Ergonomia: projeto e produção. 211 edição rev. e ampl. - São Paulo: Edgard Blücher, 2005.

KARAHAN, A. et al. Low back pain: prevalence and associated risk factors among hospital staff. Journal of Advanced Nursing. Vol. 65, n. 3, p. 516-524, 2009.

LEVY, Y.; ELLIS, T.J. A system approach to conduct an effective literature review in support of information systems research. Informing Science Journal, v.9, p.181-212, 2006. 
LIMA, M. C.T.F.C. Análise das condições ergonômicas da situação de trabalho dos auxiliares de enfermagem em uma unidade de internação hospitalar. Dissertação (Mestrado em Engenharia) - Escola de Engenharia. Universidade Federal do Rio Grande do Sul. Porto Alegre, 2004.

LOOZE, M.P. et al. Effec of individually chosen bed-height adjustments on the lowback stress of nurses. Scandinavian Journal of Work Environmental Health. 20 (6): 427- 434, 1994.

MAGNANO, T.S.B.S. et al. Condições de trabalho, características sócio-demográficas e distúrbios musculoesqueléticos em trabalhadores de enfermagem. Acta Paulista de Enfermagem, v. 23, n. 2, p. 187-93, mar.-abr. 2010.

MARIA, V.; CARDOSO, B. Ergonomia Hospitalar: Atividades de Alimentação e Deficiência dos Equipamentos Disponíveis. Revista Produção, Associação Brasileira de Engenharia de Produção - ABEPRO, 1998. Disponível em: <www.abepro.org.br/biblioteca/enegep1998_art353.pdf>. Acessado em: 02/04/14

MARZIALE, M.H.P.; ROBAZZI, M.L.C.C. 0 trabalho de enfermagem e a ergonomia. Revista Latino-Americana de Enfermagem, v. 8, n. 6, p. 124-7, dez. 2000.

Ministério da saúde. Instrução normativa INSS/DC n 98, de 05 de dezembro de 2003. Aprova Norma Técnica sobre lesões por Esforços Repetitivos - LER ou Distúrbios Osteomusculares Relacionados ao Trabalho - DORT. Brasília: Ministério da Saúde; 2003.

NELSON, A. \& BAPTISTE, A. S. Evidence-based practices for safe patient handling and movement. Clinical Reviews in Bone and Mineral Metabolism. Vol. 4, n. 1, p. 55-69, 2006.

OWEN, B.D. Preventing injuries using an ergonomic approach. AORN Journal, Thorofare (NJ), Dec; 72(6):1031-6, 2000.

PARADA; E.O. et al. Lesões ocupacionais afetando a coluna vertebral em trabalhadores de enfermagem. Rev. Latino-am Enfermagem. Janeiro-fevereiro; 10(1):64-9, 2002.

RADOVANOVIC, C.A.T.; ALEXANDRE N.M.C. Desenvolvimento de um instrumento para avaliar a movimentação e transferência de pacientes: um enfoque ergonômico. Rev. Esc. Enferm. USP.36: 231-9, 2002.

RODRIGUES; L. H. et al. Problemas ergonômicos enfrentados por profissionais de enfermagem em ambientes hospitalares: aspectos da interface trabalhadores $x$ equipamentos. Cadernos de estudos tecnológicos, v. 01, n. 01, julho, 2013. 
ROSSI, C.G.; et al. Aspectos ergonômicos na transferência de pacientes : um estudo realizado com trabalhadores de uma central de transportes de um hospital universitário. Rev Esc Enferm USP, v. 35, n. 3, p. 249-256, 2001.

STORDEUR, S.; et all. Leadership, organizational stress, and emotional exhaustion among hospital nursing staff. J. Adv. Nurs. 35: 533-42, 2001.

VOLPI, Sylvia. A avaliação ergonômica. s.n.t. Disponível em: http:// http://www.sylviavolpi.com.br/artigos/artigo_07.htm. Acesso em: 30/03/14

ZANON, E.; MARZIALE, M. H. P. Avaliação da postura corporal dos trabalhadores de enfermagem na movimentação de pacientes acamados. Rev. Esc. Enf. USP, v. 34, n. 1, p. 26-36, mar. 2000. 